\title{
Diseño de una losa de hormigón liviano de poliestireno expandido reciclado modificado
}

Design of a modified recycled expanded styrene lightweight concrete slab

\author{
Gregorio Marcelo Cabezas \\ ediuciv@gmail.com \\ Universidad Autónoma Tomás Frías, Bolivia
}

Artículo recibido enero 2019 | Arbitrado en feb-marzo 2019 | Publicado en mayo 2019

\section{RESUMEN}

La búsqueda de la reducción de la carga muerta de una losa de hormigón, mediante la utilización del hormigón liviano, permite la reducción del tamaño de columnas, zapatas y otros elementos de cargas en las cimentaciones particularmente. En el presente estudio se diseña un hormigón estructural con agregado liviano reciclado, reemplazando parte del agregado grueso de una mezcla tradicional por Poliestireno reciclado, previo tratamiento térmico para llegar a estándares óptimos de calidad de acuerdo a lo establecido en el código (ACI Committee 318. y American Concrete Institute, 2014) para hormigón estructural liviano. Mediante el estudio se concluyó que el sistema cumple con criterios del código (ACI Committee 318. y American Concrete Institute., 2014) disminuyendo un $26,80 \%$ el peso propio de la losa en comparación a la de un hormigón convencional, con resistencia más que satisfactoria para cargas de viviendas familiares.

Palabras clave: Losa, hormigón liviano, poliestireno expandido

\section{ABSTRACT}

The search to reduce the dead load of a concrete slab, through the use of lightweight concrete, allows the reduction of the size of columns, footings and other load elements in foundations particularly. In the present work, a structural concrete with recycled lightweight aggregate is designed, replacing part of the coarse aggregate of a traditional mixture with recycled polystyrene, after heat treatment to reach optimal quality standards according to what is established in the code (ACI Committee 318. and American Concrete Institute, 2014) for lightweight structural concrete. Through the study, it was concluded that the system meets the code criteria (ACI Committee 318. and American Concrete Institute., 2014), reducing the slab's own weight by $26.80 \%$ compared to that of conventional concrete, with greater resistance than satisfactory for loads of family dwellings.

Key words: Slab, lightweight concrete, expanded polystyrene 


\section{INTRODUCCIÓN}

Si se observa hacia el pasado y se realiza un recorrido en la línea del tiempo hasta el presente, se revelara que el estado actual de la sociedad es el resultado de constantes innovaciones tecnológicas, la gran mayoría de ellas tendientes a optimizar recursos y en definitiva a hacer más sencillo el arte de proyectar; denotándose cambios radicales en cómo se diseñan $\mathrm{y}$ construyen los diferentes sistemas estructurales que son usados día a día.

Las losas constituyen uno de los elementos estructurales que aportan la mayor parte del peso propio a la estructura, lo que deriva en diversas limitantes de diseño y costos, esto debido a que el hormigón es en la actualidad el material más usado en la industria de la construcción, sin embargo la alta densidad o peso volumétrico de los hormigones convencionales alrededor de $2400 \mathrm{~kg}$. /m3 ha sido un inconveniente donde la carga muerta es un factor importante.

Lo anterior redunda en construcciones pesadas, vigas de gran peralte, columnas robustas y cimentaciones amplias 0 complejas. Todo esto debido al excesivo peso muerto de las losas de hormigón convencional, lo cual se traduce en un elevado costo de la obra. Se desea evaluar el desempeño estructural de una losa liviana unidireccional usando poliestireno expandido reciclado modificado en la mezcla en reemplazo del agregado grueso del hormigón convencional, con el fin de obtener condiciones aceptables de resistencia y mayores beneficios económicos al momento de diseñar.

Actualmente en América latina el uso de hormigones livianos de alto desempeño no es notorio, aunque países como Chile ya están empezando a invertir en el desarrollo de este nuevo material. (Vidal, 2010; Ascencio, 2006) Sin embargo, en países como Noruega y EE.UU. el uso de hormigón liviano de alto desempeño en obras civiles lleva años, ya que los estudios y aplicación inician mucho antes en países con mayor desarrollo de materiales en general.

Los primeros hormigones livianos utilizados para construir edificaciones surgieron en el Imperio Romano en los años 20 a.C (Hernández, 2011). Estos eran resultado de la mezcla de materiales cementantes formados a partir de limos quemados con materiales de baja densidad como lo es piedra pómez. Entre las obras realizadas con este tipo de hormigón destacan: La cúpula del Panteón de Agripa (25 a.C) y los arcos del Coliseo Romano (70 d.C), éste último con luces mayores a 25 metros (Galli, 2007).

Los avances tecnológicos han permitido la experimentación con agregados ricos en sílice como la cascarilla de arroz (Arce, 1997), agregados artificiales plásticos (poliestireno) y agregados livianos (arcillas, pizarras y escorias expandidas). Los primeros edificios construidos con hormigones estructurales livianos aparecieron luego de la primera Guerra Mundial. En el año 1922 se construyó la ampliación del Gimnasio de la Escuela de Deportes Acuáticos de la ciudad de Kansas, naciendo así la primera obra de hormigón liviano estructural de la historia. El suelo donde se cimentó dicho edificio tenía una capacidad de soporte muy baja, por esta razón se utilizó un hormigón liviano, aligerando la carga generada por el peso propio (Expanded Shale, Clay and Slate Institute, 1971). Durante el año 1928 se realizó un estudio 
de estructuración con el fin de incrementar el número de plantas del edificio de oficinas de la compañía de teléfono Southwestern Bell en la ciudad de Kansas, el cual contaba con 14 pisos. Así, se investigó sobre la cimentación y se determinó que a dicha estructura se le podía adicionar 8 pisos más utilizando hormigón convencional, no obstante al utilizar hormigón liviano en base a arcillas expandidas, dicha construcción se incrementó en 14 plantas (Hernández, 2011).

De acuerdo con el contexto donde se desarrolló el estudio surgió la interrogante sobre ¿En qué porcentaje de sustitución del árido grueso genera una menor resistencia del hormigón para elementos estructurales tipo losa, para el departamento de Potosí? Para dar respuesta y manteniendo presente el objeto de estudio acerca del análisis y diseño de una losa de hormigón liviano, se evalua el comportamiento de las losas de hormigón liviano. De esta manera, se logra la finalidad de obtener hormigón estructural con agregados livianos reciclados (MEPS) para la utilización en elementos estructurales tipo losa unidireccional. Cumpliendo principalmente con los objetivos específicos como lo son la Caracterización del árido grueso liviano para su uso en el hormigón estructural; Determinar el porciento de sustitución óptimo de árido grueso por agregado liviano, en la elaboración de hormigón liviano para elemento estructural tipo losa; Cuantificar mediante ensayos de carga la resistencia máxima del hormigón liviano y la evaluación del desempeño estructural de una losa liviana unidireccional confeccionada en base a poliestireno expandido modificado (MEPS).

De lo anterior, la necesidad de generar investigaciones sobre nuevos materiales de construcción, capaces de facilitar el diseño y construcción de estructuras, con elementos de menor densidad que no comprometan las propiedades de resistencia. Dentro de los materiales constructivos de baja densidad muy pocos satisfacen condiciones aceptables de resistencia, para ser utilizados como elementos estructurales.

$\mathrm{Al}$ ser una obligación el cuidar el medio ambiente y mucho más ahora que el planeta sufre de un calentamiento global, es mejor realizar el uso del poliestireno expandido en las distintas cosas son realizadas a diario. Se ha pensado en evitar el incineramiento de los desperdicios producidos en la línea de EPS, por medio de una empresa gestora o que vaya a parar en los botaderos de basura, es un plástico que no se degrada fácilmente; la mejor opción es poder volver a utilizarlo en el proceso, se podría pensar en un proyecto complementario de gestión ambiental para la reutilización del EPS que se utilizará como agregado liviano.

En el caso de Bolivia, se estima que 0,5 kilos de residuos son generados por habitante cada día, lo cual equivale a 4.782 toneladas de residuos sólidos por día o $1^{\prime} 745.280$ toneladas por año, de los cuales el $87 \%$ se generan en áreas urbanas. Se estima que el $55,2 \%$ de estos corresponde a residuos orgánicos, $10,2 \%$ a plásticos, $6,5 \%$ a papel y cartón, 2,9\% a vidrio, 2,5 \% a metales y $22,7 \%$ a otros. No obstante, la mayor parte ingresa a los rellenos sanitarios de los Municipios de Bolivia. El aprovechamiento de manera formal e informal de los residuos inorgánicos es el $3,70 \%$ y la recuperación del poliestireno expandido es nula en nuestro país (MMAyA, VAPSB, y DGGIRS, 2011).

MATERIALES Y METODOS

Para la realización de probetas de hormigón, con agregado de poliestireno 
expandido modificado (MEPS), se confeccionaron 36 probetas, para los cuales fueron utilizadas 24 para el ensayo a compresión, y 12 para el ensayo a tracción indirecta. El hormigón que se utilizó es el H25 estructural

\section{Cemento}

El cemento utilizado para el estudio fue un cemento IP40, producto certificado que cumple con las exigencias de la normativa Boliviana vigente NB11:12. De acuerdo a recomendación del fabricante el cemento se debe guardar en ambientes ventilados.

\section{Áridos}

Se utilizó arena cernida y gravilla seleccionada, facilitada por áridos Azángaro, limpia de impurezas de materia orgánica. El material fue analizado para obtener su banda, porcentaje de humedad, masa específica aparente, masa unitaria y su curva granulométrica, según exigencias de la normativa Boliviana vigente NB 596:16 y NB 598:91.

\section{Agua}

El agua empleada tenía las condiciones básicas, las mismas requeridas para la construcción de cualquier tipo de hormigón, por ejemplo no debe presentar materia orgánica o presencia de sustancias químicas dañinas, que produzcan oxidación en la armadura u segregación de los áridos, por ejemplo las sales o azucares. En la construcción de la mezcla se emplea agua potable de AAPOS, ya que el agua de la red pública cumple con los requisitos de $\mathrm{PH}$, dureza, sólidos en suspensión, materia orgánica exigida para el trabajo en hormigón NB 637:94

\section{Poliestireno expandido modificado}

Para este estudio el poliestireno fue recolectado de los residuos generados y remanentes de las construcciones realizadas en la ciudad de Potosí, las que luego son eliminadas en vertederos de la zona, provocando contaminación del medio ambiente.

\section{Densidades de los materiales antes y después del tratamiento térmico}

Se obtuvo 6 muestras para realizar un ensayo y definir el volumen y densidades con el fin de obtener su capacidad de soporte ver Figura 1. Los resultados obtenidos se muestran en la Tabla 1. 


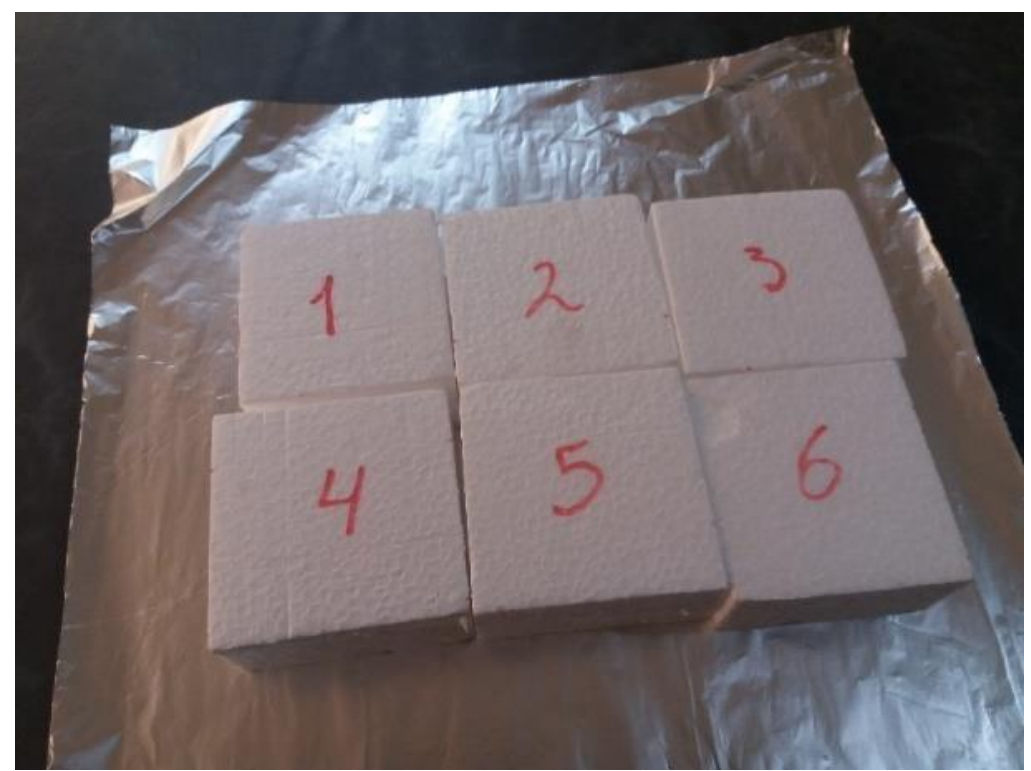

Figura 1. Muestras para ensayo (Fuente: autor)

Tabla 1 Cálculo de densidades de poliestireno recolectado

\begin{tabular}{|c|c|c|c|c|c|c|}
\hline \multirow{2}{*}{ Muestra } & \multirow{2}{*}{$\begin{array}{c}\text { peso } \\
\text { [gr] }\end{array}$} & \multicolumn{2}{|c|}{ Dimensiones en [cm] } & \multirow[b]{2}{*}{$\mathbf{h}$} & Volumen & \multirow{2}{*}{$\begin{array}{c}\text { Densidad } \\
\mathrm{kg} / \mathrm{m}^{3}\end{array}$} \\
\hline & & a1 & a2 & & {$\left[\mathrm{cm}^{3}\right]$} & \\
\hline 1 & 4,52 & 9,40 & 9,90 & 4,80 & 446,7 & 10,1 \\
\hline 2 & 5,15 & 10,00 & 10,00 & 4,80 & 480,0 & 10,7 \\
\hline 3 & 4,90 & 10,00 & 9,90 & 4,80 & 475,2 & 10,3 \\
\hline 4 & 4,84 & 10,00 & 10,00 & 4,80 & 480,0 & 10,1 \\
\hline 5 & 4,81 & 9,90 & 10,00 & 4,80 & 475,2 & 10,1 \\
\hline 6 & 5,06 & 9,50 & 10,00 & 4,80 & 456,0 & 11,1 \\
\hline & & & & & Promedi & 10,4 \\
\hline
\end{tabular}

El poliestireno expandido sin modificar tenía una densidad aproximada de $10,4 \mathrm{~kg} / \mathrm{m}^{3}$, en la Figura 2 se muestra el EPS cortado y listo para el tratamiento térmico. Una vez realizado el tratamiento térmico dentro del horno con el tiempo y temperatura de cocción utilizados ver Figura 3, fueron los determinados como óptimos en el estudio realizado por (Kan y Demirboğa, 2009) los que corresponden a una exposición de 15 minutos a $130^{\circ} \mathrm{C}$ se puede visualizar el cambio rotundo de volumen ver Figura 4. Esto alcanza aproximadamente al $2.78 \%$ del volumen original. Los resultados se muestran en la Tabla 2. 


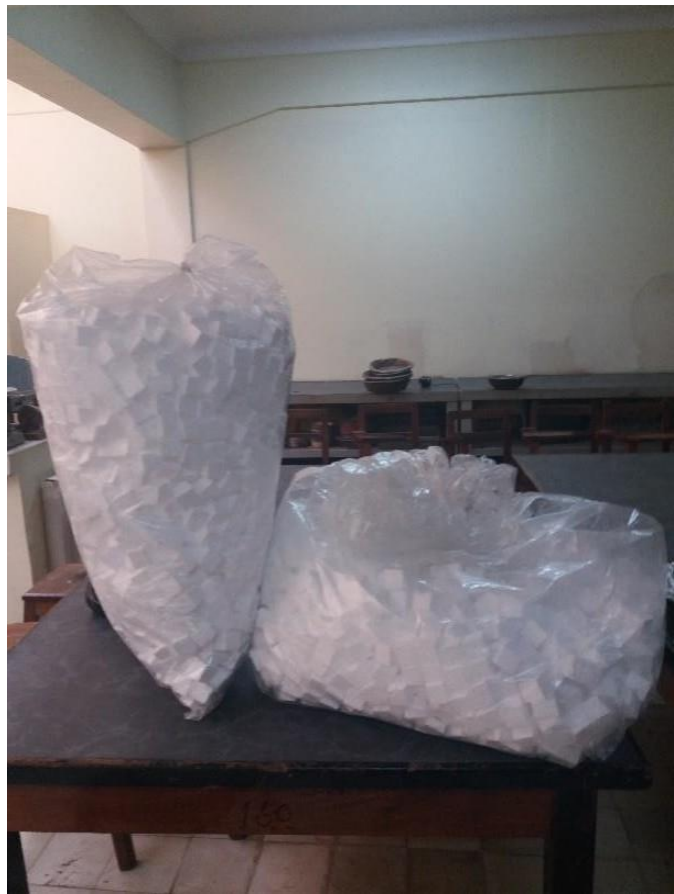

Figura 2. Poliestireno expandido antes del tratamiento térmico (Fuente: Autor)

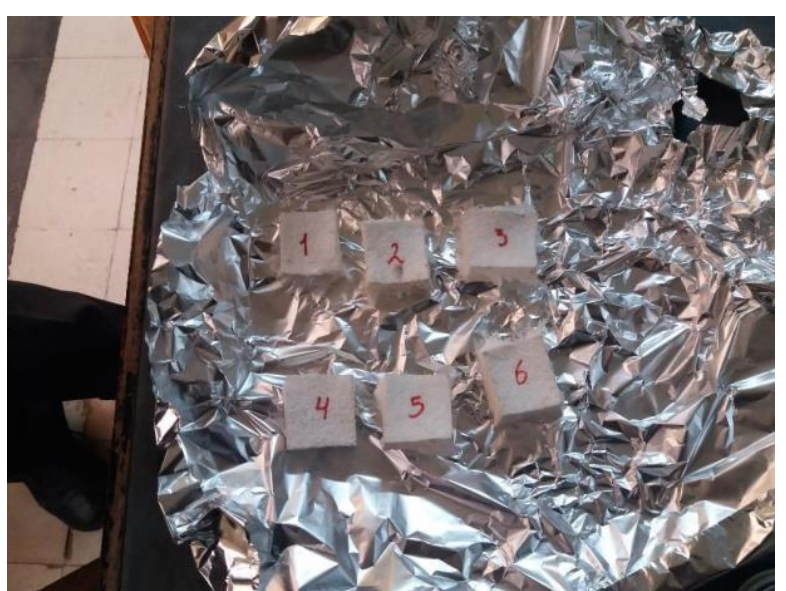

Figura 3. Poliestireno expandido al finalizar el tratamiento térmico (Fuente: Autor)
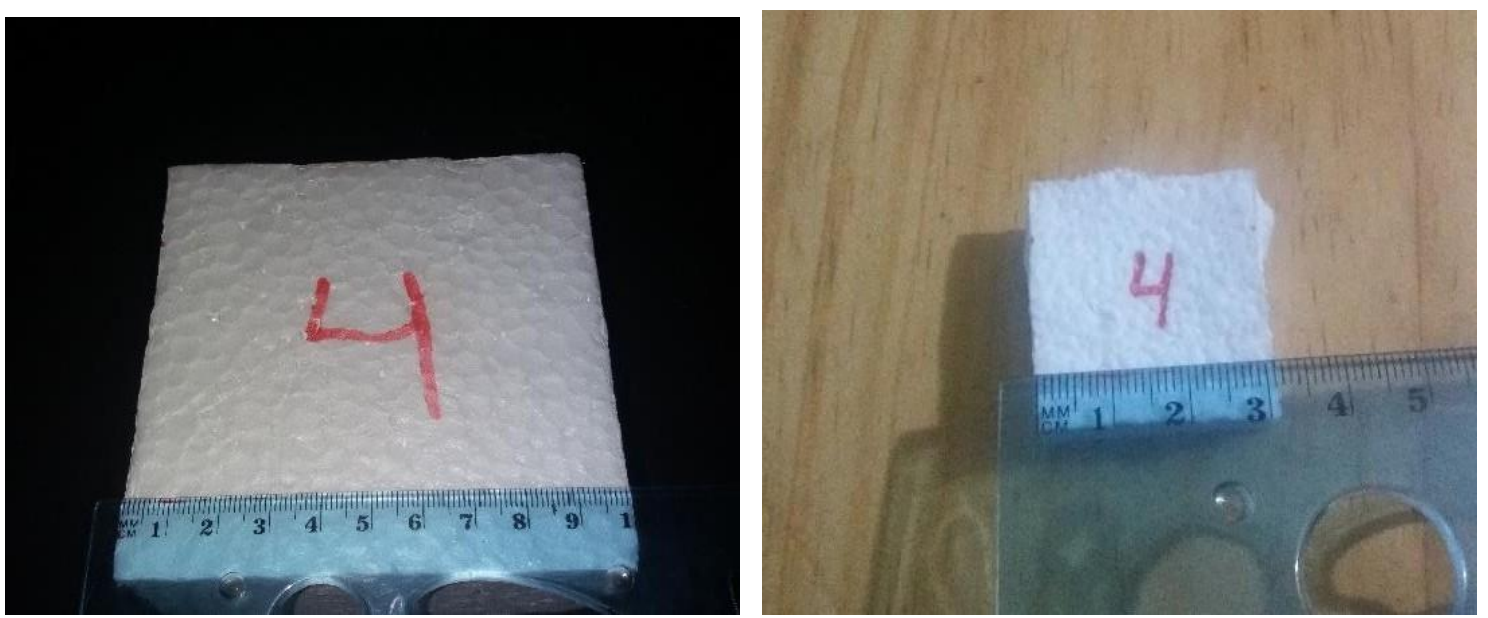

Figura 4. Diferencia de volumen después del tratamiento térmico (Fuente: autor) 
Tabla 2. Cálculo de densidades de poliestireno modificado

\begin{tabular}{|c|c|c|c|c|c|c|}
\hline \multirow{2}{*}{ Muestra } & \multirow{2}{*}{$\begin{array}{r}\text { peso } \\
\text { [gr] }\end{array}$} & \multicolumn{3}{|c|}{ Dimensiones en [cm] } & \multirow{2}{*}{$\begin{array}{c}\text { Volumen } \\
{\left[\mathrm{cm}^{3}\right]}\end{array}$} & \multirow{2}{*}{$\begin{array}{c}\text { Densidad } \\
\mathrm{kg} / \mathrm{m}^{3}\end{array}$} \\
\hline & & a1 & $\mathbf{a} 2$ & h & & \\
\hline 1 & 4,50 & 2,90 & 3,20 & 1,30 & 12,1 & 373,0 \\
\hline 2 & 5,13 & 3,10 & 3,30 & 1,20 & 12,3 & 417,9 \\
\hline 3 & 4,86 & 3,30 & 3,10 & 1,20 & 12,3 & 395,9 \\
\hline 4 & 4,82 & 3,10 & 3,30 & 1,40 & 14,3 & 336,5 \\
\hline 5 & 4,79 & 3,20 & 3,20 & 1,40 & 14,3 & 334,1 \\
\hline \multirow[t]{2}{*}{6} & 5,02 & 3,00 & 3,30 & 1,30 & 12,9 & 390,1 \\
\hline & & & & & Promedic & 374,6 \\
\hline
\end{tabular}

\section{Dosificación}

Los ensayos desarrollados en este estudio fueron realizados según la normativa boliviana vigente para hormigones $\mathrm{CBH}$ 87:87. Ya que para hormigón livianos estructurales no existe una normativa específica. Posteriormente se inició el proceso de dosificación de los hormigones a estudiar, determinando los porcentajes de sustitución de grava por MEPS, de esta forma se dejó en claro los volúmenes de cada material necesario ver Tabla 3. Se realizaron 24 probetas para el ensayo a compresión y 12 para el ensayo a tracción, en total 36 probetas para ensayar. (Tabla 4).

Tabla 3. Porcentajes de sustitución para las series

\begin{tabular}{ccc}
\hline Serie & Gravilla & MEPS \\
\hline A & $100,00 \%$ & $0,00 \%$ \\
B & $40,00 \%$ & $60,00 \%$ \\
C & $30,00 \%$ & $70,00 \%$ \\
D & $20,00 \%$ & $80,00 \%$ \\
\hline
\end{tabular}

Tabla 4. Cubicación de los materiales para 36 probetas

\begin{tabular}{cccccc}
\hline Serie & $\begin{array}{c}\text { Cemento } \\
{[\mathbf{K g}]}\end{array}$ & $\begin{array}{c}\text { Agua } \\
{[\mathbf{L}]}\end{array}$ & $\begin{array}{c}\text { Gravilla } \\
{[\mathbf{k g}]}\end{array}$ & $\begin{array}{c}\text { MEPS } \\
{[\mathbf{k g}]}\end{array}$ & $\begin{array}{c}\text { Arena } \\
{[\mathbf{k g}]}\end{array}$ \\
\hline A & 25,0 & 11,2 & 69,4 & 0,0 & 41,2 \\
B & 25,0 & 11,2 & 27,7 & 10,8 & 41,2 \\
C & 25,0 & 11,2 & 20,8 & 12,5 & 41,2 \\
D & 25,0 & 11,2 & 13,9 & 14,3 & 41,2 \\
\hline
\end{tabular}




\section{Confección de probetas}

Debido a su baja densidad y tendencia a flotar, los MEPS fueron vertidos en primero en la mezcladora. El agua se vertió en dos partes, se agregó la arena, el cemento, el material granular y para finalizar el resto de agua. A continuación la mezcladora trabajó en intervalos de 15 a 20 segundos para evitar que los MEPS subieran a la superficie por ser un material más liviano que la gravilla utilizada ver Figura 5, después se vació a las probetas, previamente revestidos con aceite de motor.

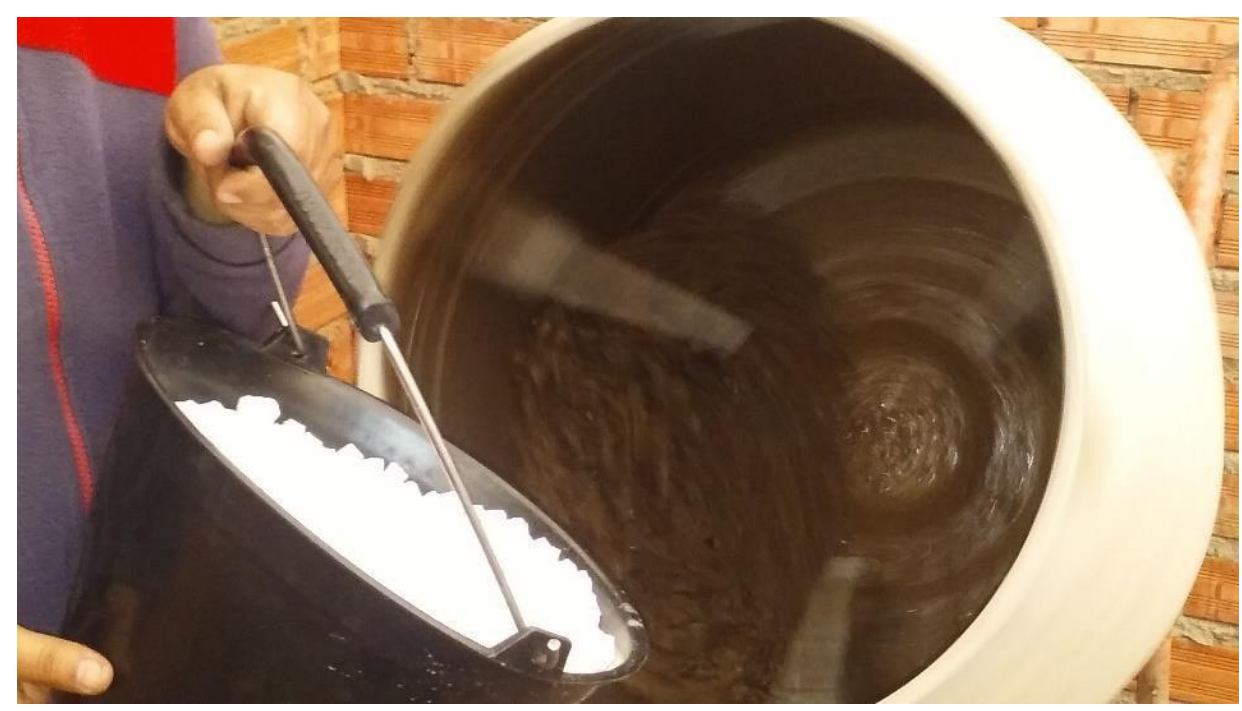

Figura 5. Mezclado del hormigón liviano (Fuente: Autor)

El desmolde de las probetas fue efectuado a las 24 horas después de ser hormigonadas según la norma Boliviana ver Figura 6.

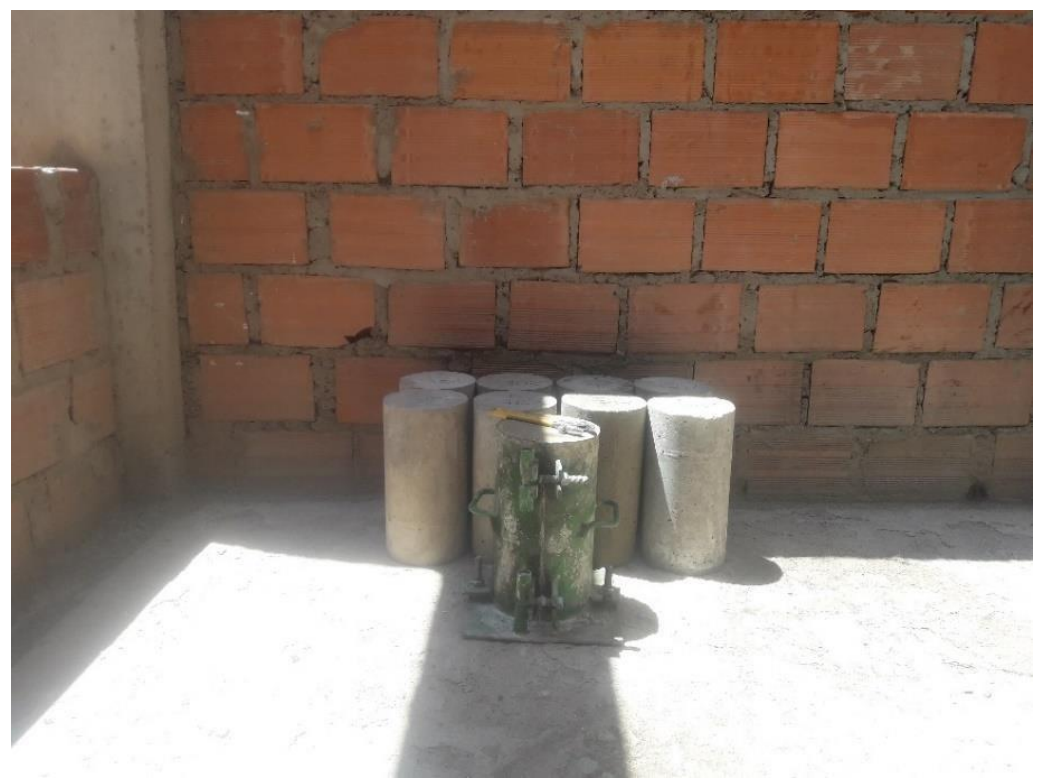

Figura 6. Desmoldado de probetas (Fuente: Autor) 
Para finalizar el proceso las probetas fueron sumergidas en agua para el curado respectivo previo marcado de las probetas especificando el caso a la cual corresponde ver Figura 7.

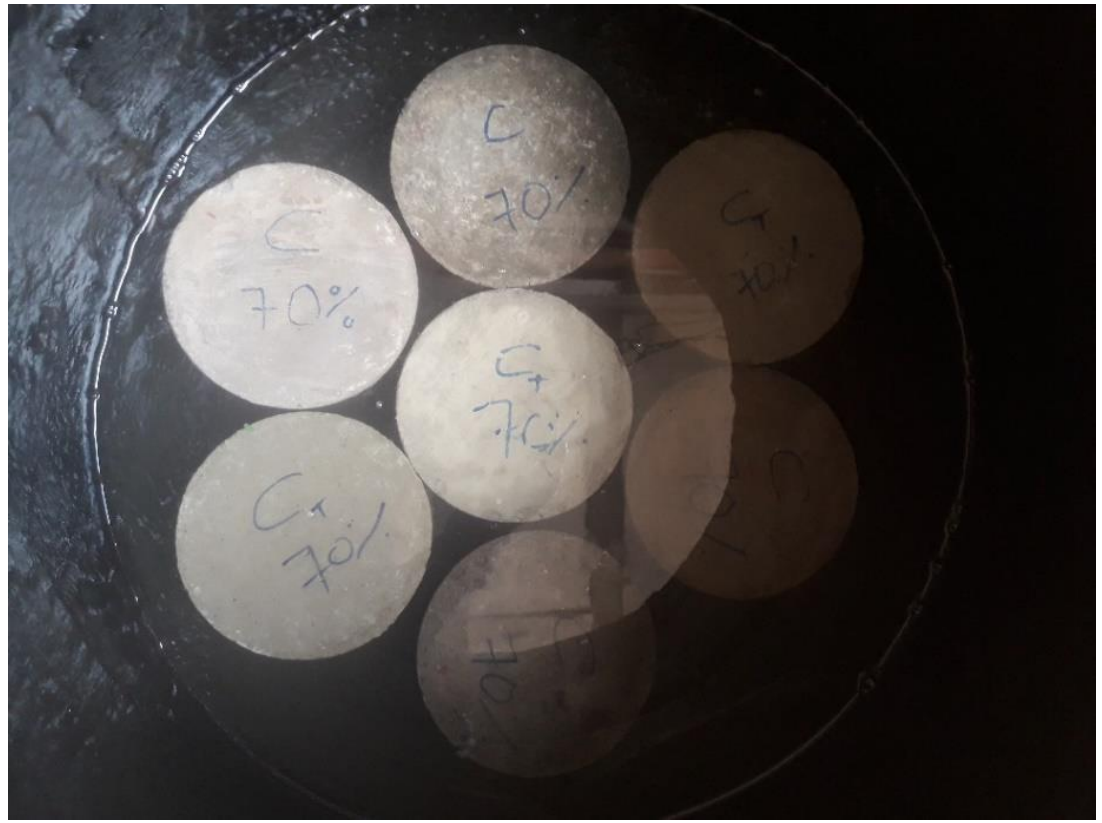

Figura 7. Curado de probetas (fuente: Autor)

Ensayo de resistencia a compresión axial

La principal característica mecánica del concreto, es la resistencia a la compresión axial, debido a que es frecuentemente utilizada en el cálculo para diseño de elementos estructurales. Este ensayo fue realizado siguiendo la norma CBH 87:87 y ASTM C39:18 ver Figura 8.

El objetivo fue determinar la resistencia máxima a la compresión de la probeta de hormigón estudiado frente a una carga axialmente aplicada.

La resistencia a compresión se calcula por medio de la siguiente ecuación:

$$
f_{c}=\frac{P}{A}
$$

Dónde: $\mathrm{P}=$ carga de rotura determinada en el ensayo $\mathrm{A}=$ área transversal de la probeta. 


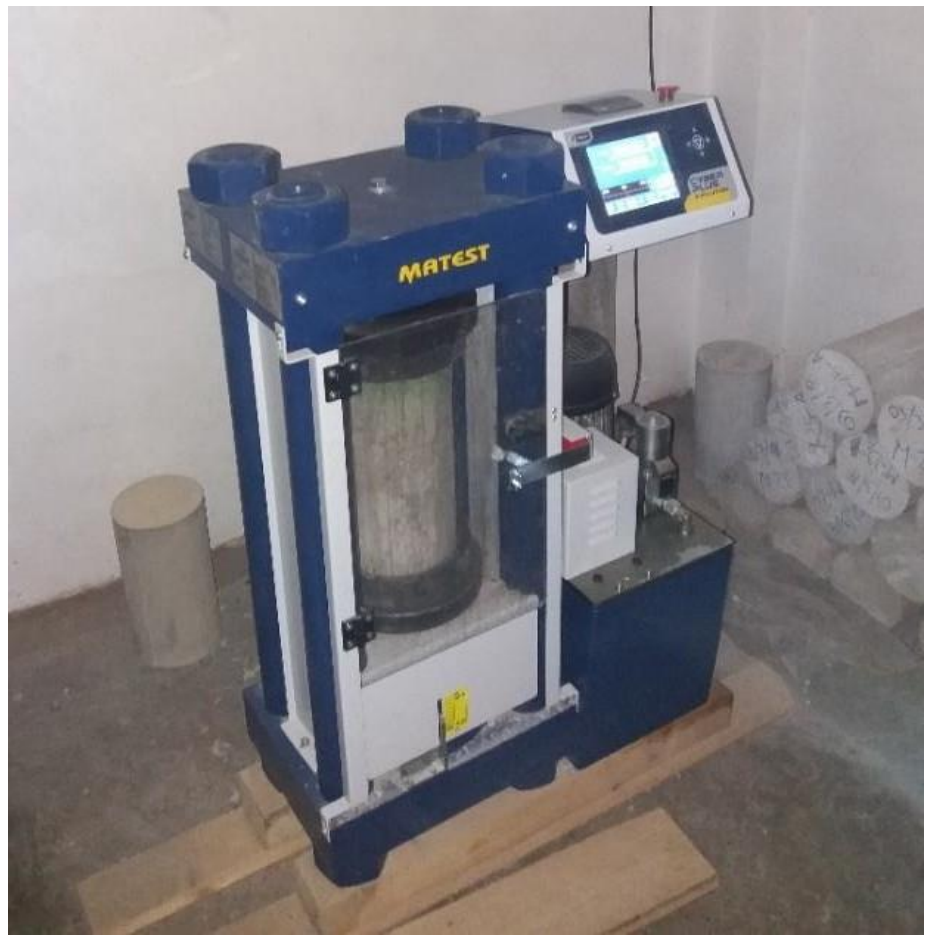

Figura 8. Ensayo a compresión axial (Fuente: Autor)

\section{Ensayo de resistencia a tracción indirecta}

La resistencia a tracción indirecta del concreto se realizó mediante el ensayo brasileño según NB 641:91 y ASTM C496:17 ver Figura 2.10, consistente en el hendimiento de las probetas cilíndricas representantes.

La finalidad de este ensayo fue determinar el esfuerzo de tracción de la probeta de hormigón a través de la aplicación de una carga lineal de compresión sobre un diámetro del disco del espécimen a ensayar.

La resistencia a tracción indirecta se calcula por medio de la siguiente ecuación:

$$
T=\begin{aligned}
& 2 P \\
& \pi l d
\end{aligned}
$$

Dónde: $\mathrm{P}=$ carga de rotura determinada en el ensayo $\mathrm{l}=$ longitud de la probeta cilíndrica

$\mathrm{d}$ = diámetro de la probeta cilíndrica 


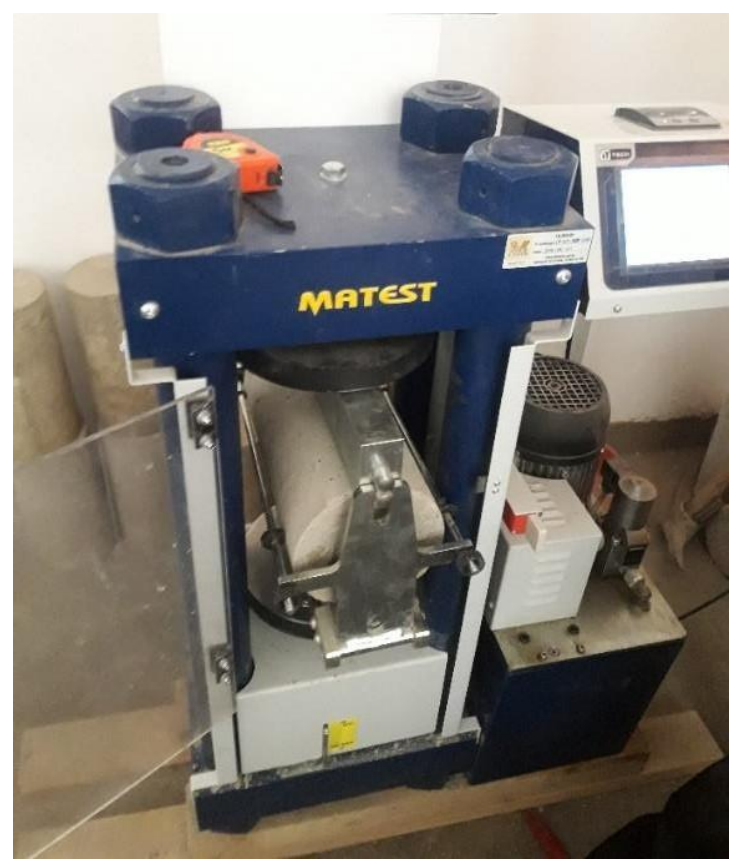

Figura 9. Ensayo a tracción indirecta (Fuente: Autor)

\section{RESULTADOS}

El poliestireno expandido modificado, posee un gran potencial para ser utilizado como agregado liviano para hormigones estructurales livianos, a través de un estudio más detallado se pueden conocer aún más sus propiedades. El límite para obtener un hormigón liviano estructural, de acuerdo al estudio realizado, se define cercana a un $70 \%$ de poliestireno expandido modificado en reemplazo de material granular.
A lo largo de los años se ha tratado de reducir el peso de los entrepisos, mediante la utilización de hormigones livianos con agregados livianos en reemplazo del agregado grueso con características tensodeformacional aceptables.

\section{Resistencia a compresión axial}

Este ensayo fue realizado siguiendo la norma CBH 87:87 y ASTM C39-18 ver Tabla 5. 
Tabla 5. Resultados finales de los ensayos a compresión

\begin{tabular}{|c|c|c|c|c|c|c|c|c|}
\hline Series & $\begin{array}{c}\text { Ensayo } \\
\text { [Días] }\end{array}$ & $\begin{array}{c}\text { Carga } \\
{[\mathrm{kN}]}\end{array}$ & $\begin{array}{c}\text { Área } \\
{\left[\mathrm{mm}^{2}\right]}\end{array}$ & $\begin{array}{l}\text { Resistencia } \\
\text { [MPa] }\end{array}$ & $\begin{array}{l}\text { Peso } \\
{[\mathrm{Kg}]}\end{array}$ & $\begin{array}{c}\text { Volumen } \\
{\left[\mathrm{cm}^{3}\right]}\end{array}$ & $\begin{array}{l}\text { Densidad } \\
{\left[\mathrm{kg} / \mathrm{m}^{3}\right]}\end{array}$ & $\begin{array}{l}\text { Densidad } \\
\text { promedio }\end{array}$ \\
\hline \multirow[t]{2}{*}{ A } & 7 & 314,61 & 17671,46 & 17,80 & 12,96 & 5301,44 & 2444,62 & 2454,68 \\
\hline & 28 & 452,57 & 17671,46 & 25,61 & 13,07 & 5301,44 & 2464,74 & \\
\hline \multirow[t]{2}{*}{ B } & 7 & 222,16 & 17671,46 & 12,57 & 9,84 & 5301,44 & 1856,73 & 1854,53 \\
\hline & 28 & 311,61 & 17671,46 & 17,63 & 9,82 & 5301,44 & 1852,33 & \\
\hline \multirow[t]{2}{*}{$\mathrm{C}$} & 7 & 202,04 & 17671,46 & 11,43 & 9,00 & 5301,44 & 1698,28 & 1756,76 \\
\hline & 28 & 304,81 & 17671,46 & 17,25 & 9,62 & 5301,44 & 1815,23 & \\
\hline \multirow[t]{2}{*}{ D } & 7 & 176,34 & 17671,46 & 9,98 & 8,72 & 5301,44 & 1644,84 & 1710,86 \\
\hline & 28 & 290,28 & 17671,46 & 16,43 & 9,42 & 5301,44 & 1776,88 & \\
\hline
\end{tabular}

\section{Resistencia a tracción indirecta}

Este ensayo fue realizado según CBH 641:1991 y ASTM C496:17 ver Tabla 6.

Tabla 6. Resultados finales de los ensayos a tracción indirecta

\begin{tabular}{ccccccccc}
\hline Serie & $\begin{array}{c}\text { Ensayo } \\
{[\text { Días] }}\end{array}$ & $\begin{array}{c}\text { Carga } \\
{[\mathbf{k N}]}\end{array}$ & $\begin{array}{c}\mathbf{l} \\
{[\mathbf{m m}]}\end{array}$ & $\begin{array}{c}\mathbf{d} \\
{[\mathbf{m m}]}\end{array}$ & $\begin{array}{c}\text { Resistencia } \\
{[\mathbf{M P a}]}\end{array}$ & $\begin{array}{c}\text { Peso } \\
{[\mathbf{K g}]}\end{array}$ & $\begin{array}{c}\text { Volumen Densidad } \\
{\left[\mathbf{c m}^{3}\right]}\end{array}$ & {$\left[\mathbf{k g} / \mathbf{m}^{3}\right]$} \\
\hline A & 28 & 183,13 & 300 & 150 & 2,59 & 12,80 & 5301,44 & 2414,44 \\
B & 28 & 129,81 & 300 & 150 & 1,84 & 9,61 & 5301,44 & 1812,72 \\
C & 28 & 124,19 & 300 & 150 & 1,76 & 9,10 & 5301,44 & 1717,14 \\
D & 28 & 118,93 & 300 & 150 & 1,68 & 8,93 & 5301,44 & 1685,08 \\
\hline
\end{tabular}

\section{Reducción de la densidad y resistencia de compresión axial}

La tabla 7 presenta los resultados comparativos, de los porcentajes de reducciones de la densidad de las mezclas realizadas, y los porcentajes de reducción de la resistencia a compresión axial.

Tabla 7. Reducción de densidad y resistencia a compresión axial

\begin{tabular}{cccccc}
\hline Serie & $\begin{array}{c}\text { Ensayo } \\
\text { [Días] }\end{array}$ & $\begin{array}{c}\text { Densidad } \\
{\left[\mathbf{k g} / \mathbf{m}^{3} \text { ] }\right.}\end{array}$ & $\begin{array}{c}\text { Reducción } \\
\text { Densidad [\%] }\end{array}$ & $\begin{array}{c}\text { Resistencia } \\
\text { [MPa] }\end{array}$ & $\begin{array}{c}\text { Reducción } \\
\text { Resistencia [\%] }\end{array}$ \\
\hline A & 7 & 2444,62 & & 17,80 & \\
& 28 & 2464,74 & & 25,61 & \\
B & 7 & 1856,73 & $24,0 \%$ & 12,57 & $29,4 \%$ \\
& 28 & 1852,33 & $24,8 \%$ & 17,63 & $31,1 \%$ \\
C & 7 & 1698,28 & $30,5 \%$ & 11,43 & $35,8 \%$ \\
& 28 & 1815,23 & $26,4 \%$ & 17,25 & $32,6 \%$ \\
D & 7 & 1644,84 & $32,7 \%$ & 9,98 & $43,9 \%$ \\
& 28 & 1776,88 & $27,9 \%$ & 16,43 & $35,9 \%$ \\
\hline
\end{tabular}

\section{Reducción de la resistencia a tracción indirecta}

La tabla 8 presenta los resultados comparativos, de los porcentajes de reducciones de la resistencia a tracción indirecta. 
Tabla 8. Reducción de densidad y resistencia a compresión axial

\begin{tabular}{cccc}
\hline Serie & $\begin{array}{c}\text { Ensayo } \\
\text { [Días] }\end{array}$ & $\begin{array}{c}\text { Resistencia } \\
\text { [MPa] }\end{array}$ & $\begin{array}{c}\text { Reducción } \\
\text { Resistencia [\%] }\end{array}$ \\
\hline A & 28 & 2,59 & \\
B & 28 & 1,84 & $29,1 \%$ \\
C & 28 & 1,76 & $32,2 \%$ \\
D & 28 & 1,68 & $35,1 \%$ \\
\hline
\end{tabular}

\section{Evaluación de los MEPS}

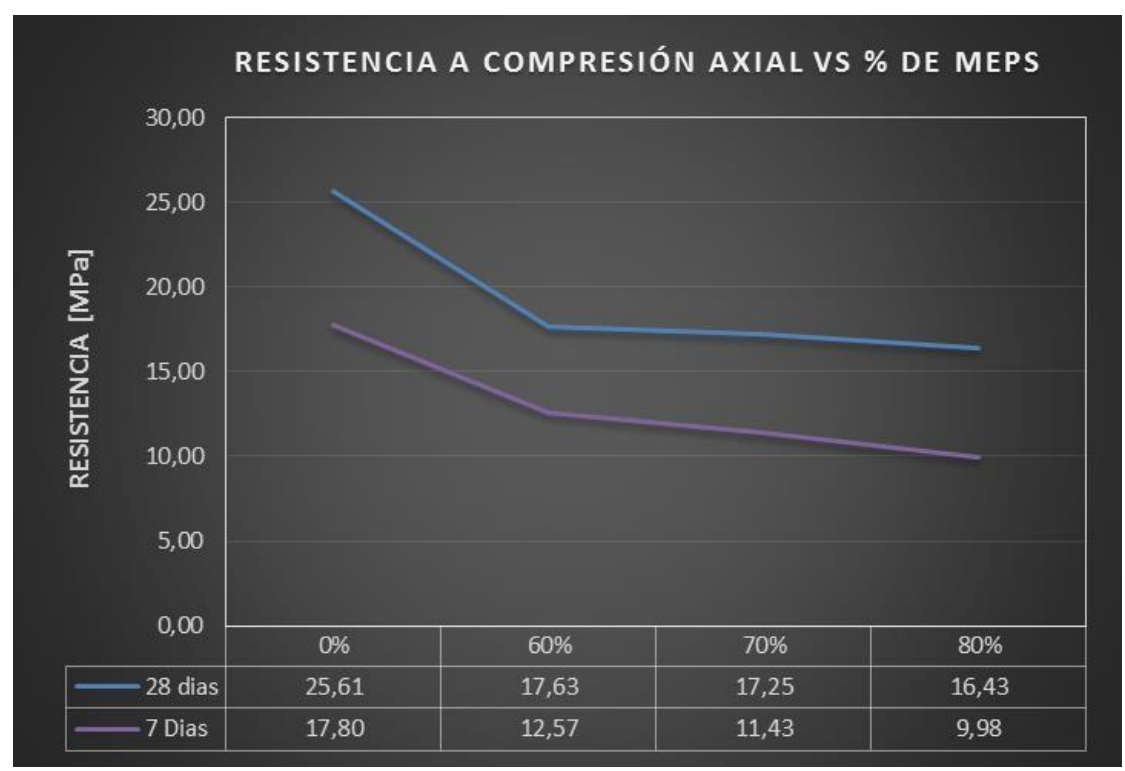

Figura 10. Resistencia a compresión axial con respecto a la cantidad de MEPS

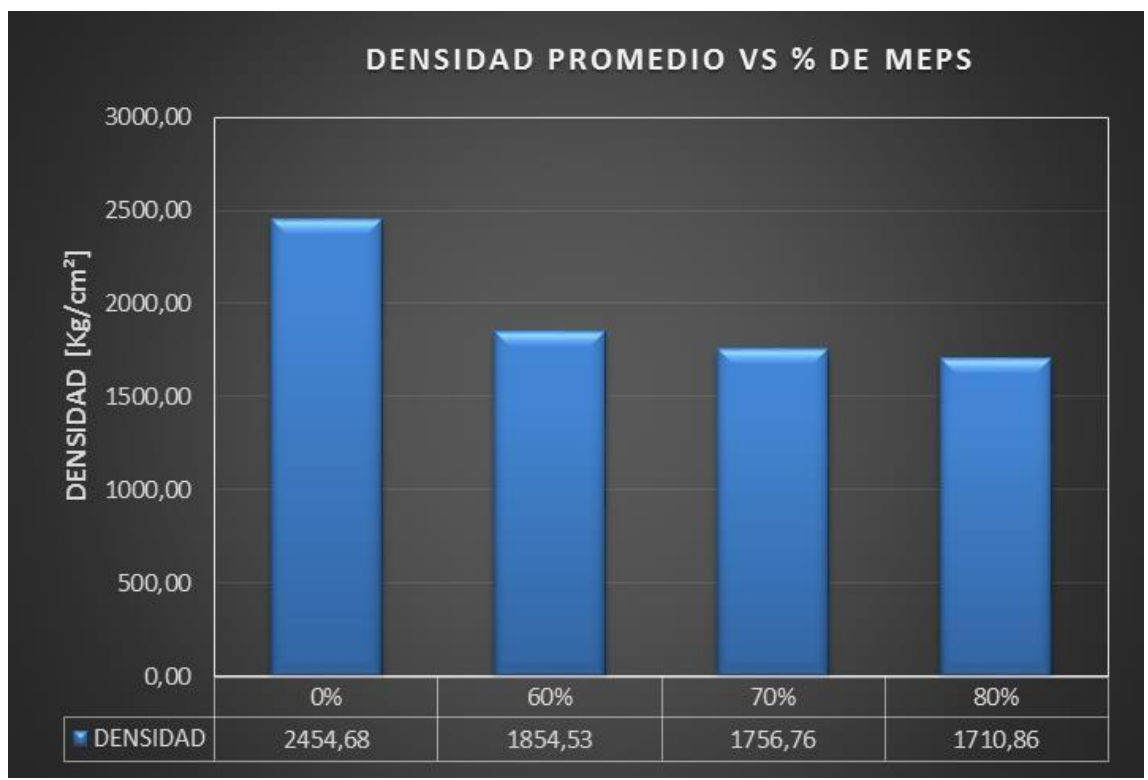

Figura 11. Densidad con respecto a la cantidad de MEPS 


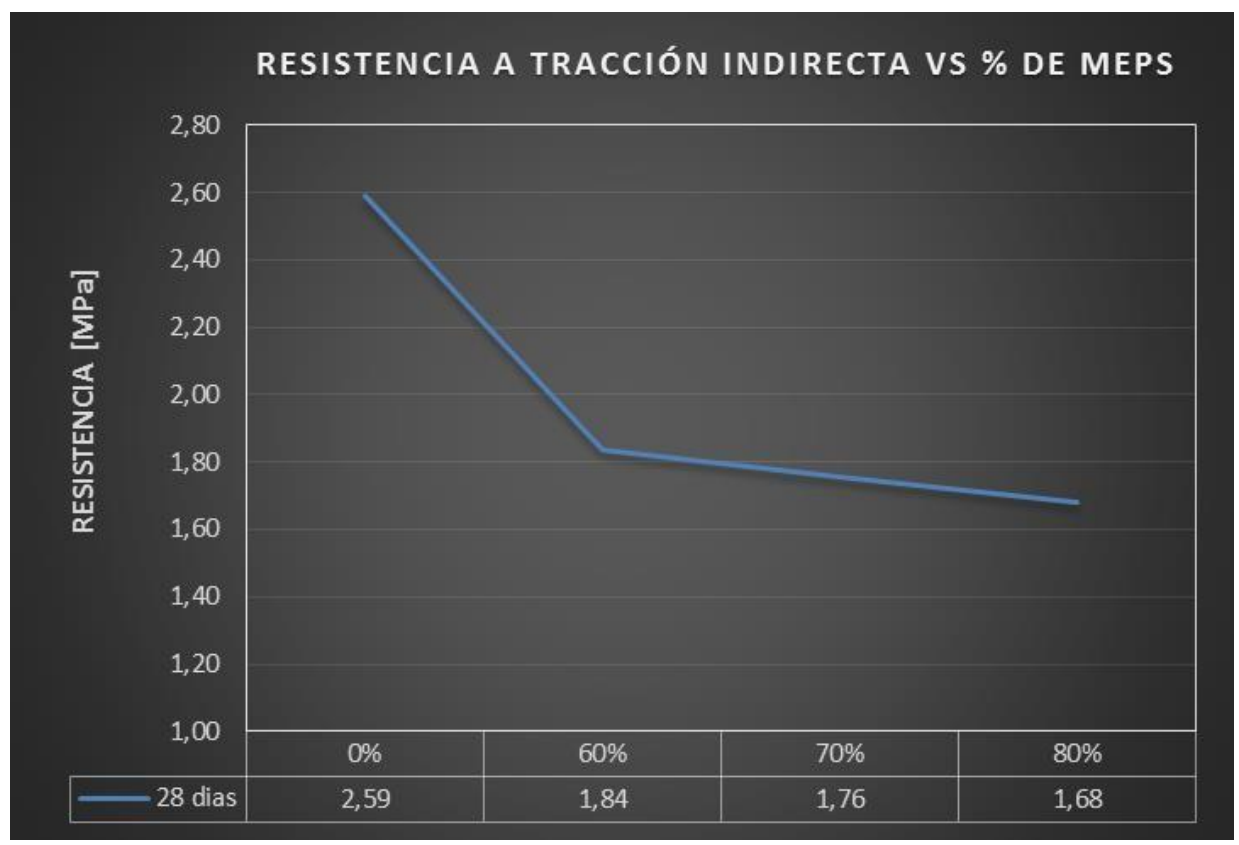

Figura 12. Resistencia a tracción indirecta con respecto a la cantidad de MEPS

En la tabla 5 del ensayo a los 28 días para la serie $\mathrm{C}$, que es conformada con un reemplazo del $70 \%$ del volumen total de gravilla, esta cumple con los estándar de calidad para ser calificado como hormigón estructural liviano al tener una resistencia a la compresión > 17 Mpa y una densidad inferior a $1800 \mathrm{~kg} / \mathrm{m}^{3}$.

Para el caso de la serie B esta cumple con la resistencia pero al ser su densidad mayor a $1800 \mathrm{~kg} / \mathrm{m}^{3}$ no puede calificarse como estructural.

Se demuestra según este trabajo y estudios anteriores que el poliestireno expandido modificado MEPS cumple con la norma y ser considerado como agregado liviano para crear hormigón estructural liviano, por sus cualidades tanto en capacidad de soporte, peso $y$ trabajabilidad.

La granulometría del poliestireno expandido modificado, permite una buena adherencia al hormigón, sin presencia de desprendimientos, segregación o una mala distribución dentro del hormigón ya endurecido.

La resistencia a la compresión reduce a medida que se incrementa más MEPS, en promedio un $33,2 \%$ con respecto a la dosificación tipo H-25 utilizada. La cual se muestra a continuación.

- Para la dosificación B con $60 \%$ de MEPS está reduce un $31,1 \%$ de resistencia.

- Para la dosificación C con $70 \%$ de MEPS está reduce un $32,6 \%$ de resistencia.

- Para la dosificación D con $80 \%$ de MEPS está reduce un 35,9\% de resistencia.

La resistencia a tracción indirecta reduce a medida que se incrementa más MEPS, en promedio un $32,1 \%$ con respecto a la dosificación tipo H-25 utilizada. La cual se muestra a continuación. 
- Para la dosificación B con $60 \%$ de MEPS está reduce un 29,1\% de resistencia.

- Para la dosificación C con $70 \%$ de MEPS está reduce un 32,2\% deresistencia.

- Para la dosificación D con $80 \%$ de MEPS está reduce un 35,1\% deresistencia.

Para la dosificación C con un $70 \%$ de MEPS se determinó según los ensayos en el laboratorio que cumple con la resistencia compresión de 17,25 Mpa y una resistencia a la tracción de 1,76 Mpa, con una densidad inferior a $1800 \mathrm{~kg} / \mathrm{m}^{3}$.

El reemplazo del agregado grueso por poliestireno expandido modificado, produjo una reducción en las densidades muy considerables, obteniendo un hormigón liviano estructural.

El poliestireno expandido modificado, posee un gran potencial para ser utilizado como agregado liviano para hormigones estructurales livianos, a través de un estudio más detallado se pueden conocer aún más sus propiedades.

El límite para obtener un hormigón liviano estructural, de acuerdo al estudio realizado, se define cercana a un $70 \%$ de poliestireno expandido modificado en reemplazo de material granular. A lo largo de los años se ha tratado de reducir el peso de los entrepisos, mediante la utilización de hormigones livianos con agregados livianos en reemplazo del agregado grueso con características tensodeformacional aceptables

El comportamiento de losas unidireccionales con hormigón liviano estructural en base a MEPS, cumple con las normativas de diseño siendo una opción viable desde una perspectiva técnica y ambiental.

Desde una perspectiva de resistencia, el elemento en estudio superó el valor predicho por las estimaciones, llegando a soportar cargas tres veces mayores a las requeridas por el diseño, concluyéndose su gran capacidad para soportar cargas, satisfaciendo así necesidades de viviendas familiares.

\section{CONCLUSIONES}

Se demuestra que el poliestireno expandido modificado MEPS cumple con la norma y ser considerado como agregado liviano para crear hormigón estructural liviano, por sus cualidades tanto en capacidad de soporte, adherencia al hormigón, peso, trabajabilidad, sin presencia de desprendimientos, segregación o una mala distribución dentro del hormigón ya endurecido.

El porcentaje de sustitución óptimo para obtener un hormigón liviano estructural, con la dosificación estudiada, puede definirse cercana a un $70 \%$ de poliestireno expandido modificado en reemplazo del árido grueso.

Según las series ensayadas, se puede concluir que se obtuvo un hormigón liviano estructural, con poliestireno expandido modificado, alcanzándose una resistencia a la compresión de 17,25 Mpa. y una resistencia a la tracción indirecta de 1,76 Mpa. La densidad obtenida es de 1756,76 $\mathrm{Kg} / \mathrm{m} 3$ es un $26,80 \%$ menor que la normalmente utilizada en elementos estructurales y las resistencias obtenidas todas superan el valor predicho por las estimaciones, por lo que el hormigón en estudio puede utilizarse con las normas, recomendaciones y métodos utilizados para el diseño de elementos estructurales de 
hormigón con las características de peso normal.

El desempeño de la losa unidireccional de hormigón liviano es comparable a la de una de hormigón tradicional, cumpliendo exitosamente con normativas, factores de carga y deformaciones admisibles. Por esta razón, el hormigón en estudio puede diseñarse de forma eficaz con las normas, recomendaciones y métodos utilizados para el diseño de elementos estructurales de hormigón con las características de peso normal.

Se permiten afirmar que la aplicación del método de diseño de losas unidireccionales de hormigón liviano con la sustitución del árido grueso estudiada cumple con la normativa de diseño en cuanto a su resistencia, siendo una opción viable desde una perspectiva técnica $\mathrm{y}$ ambiental.

\section{REFERENCIAS}

Arce, X. (1997). Hormigones livianos. (Tesis), Escuela Superior Politécnica del Litoral

ASTM C 39-18. (2018). Standard Test Method for Compressive Strength of Cylindrical Concrete Specimens

ASTM C 330-17a. (2017). Standard Specification for Lightweight Aggregates for Structural Concrete

ASTM C 496-17. (2017). Standard Test Method for Splitting Tensile Strength of Cylindrical Concrete Specimens

Ascencio, J. (2006). Diseño de losas colaborantes unidireccionales de hormigón usando planchas de zincalum y sus analogías con el uso de placas de acero galvanizado instapanel PV6-R. (Tesis), Universidad Austral de Chile

CBH 87 Comite 12.25., e Instituto Boliviano de Normalización y Calidad. (1987). Norma Boliviana del Hormigón
Armado Ministerio de Urbanismo y Vivienda.

Galli, A. (2007). Las inmigraciones italianas y su aporte técnico ornamental a la arquitectura y Urbanismo. Tesis. Buenos Aires, Argentina. Universidad de Belgrano. Pág. 22

Kan, A., y Demirboğa, R. (2009). A new technique of processing for wasteexpanded polystyrene foams as aggregates. Journal of Materials Processing Technology, 209(6), 29943000. Doi: 10.1016/j.jmatprotec.2008.07.017

Hernández, A. (2011). Comportamiento estructural de elementos flexurales de hormigón liviano. (Tesis), Pontificia Universidad Católica de Chile

MMAyA, VAPSB, y DGGIRS. (2011). Diagnóstico de la Gestión de Residuos Sólidos en Bolivia. Volumen 1, Pág. 215-218

NB 11 comité 12.1., e Instituto Boliviano de Normalización y Calidad. (2012). Cemento

Definiciones, clasificación y especificaciones: Instituto Boliviano de Normalización y Calidad

NB 596 comité 12.3., e Instituto Boliviano de Normalización y Calidad. (2016). Áridos para morteros y hormigones Requisitos: Instituto Boliviano de Normalización y Calidad

NB 598 comité 12.3., e lnstituto Boliviano de Normalización y Calidad. (1991). Áridos para morteros y hormigones Granulometría: lnstituto Boliviano de Normalización y Calidad

NB 608 comité 12.3., e lnstituto Boliviano de Normalización y Calidad. (1991). Áridos para morteros y hormigones Determinación del peso unitario: Instituto Boliviano de Normalización y Calidad

NB 637 comité 12.5., e lnstituto Boliviano de Normalización y Calidad. (1994). Agua para morteros y hormigones Requisitos: Instituto Boliviano de Normalización $\quad y \quad$ Calidad 
Vidal, F. (2010). Caracterización y evaluación del comportamiento de hormigones livianos, usando como materia prima poliestireno expandido modificado (MEPS). (Tesis), Universidad Austral de Chile 